\title{
Use of the modified Insall Salvati method for determination of vertical patellar position in dogs with and without cranial cruciate ligament rupture considering the morphology of the cranio-proximal tibia
}

\author{
Dávid Kňazovický, Valent Ledecký, Marián Hluchý, Marek Ďurej
}

University of Veterinary Medicine and Pharmacy in Košice, Small Animal Clinic, Department of Surgery, Orthopaedics, Radiology and Reproduction, Košice, Slovak Republic

Received April 3, 2012

Accepted September 19, 2012

\begin{abstract}
The aim of this study was to evaluate if the modified Insall Salvati (IS) method can be applied for the canine patient despite differences of proximal tibial morphology, and if such potential differences are also seen in dogs with cranial cruciate ligament rupture. Insall Salvati method is a simple and convenient method for determination of the vertical position of patella, by dividing the length of patella by length of the patellar ligament. The influence of the variable proximal tibial morphology on the modified (IS) index was measured and the value of modified IS index in healthy dogs $(n=25)$ was compared with dogs with cranial cruciate ligament rupture $(n=26)$. Medio-lateral radiographs of 102 stifles were evaluated. Patellar ligament length (PLL), patellar length (PL), modified IS ratio and the height of insertion point of patellar ligament on tibial tuberosity were measured. Data of the IS ratio were compared with the value of the height of the insertion point with no significant difference $(P=0.36)$. There was no significant difference $(P$ $=0.07)$ in the PLL:PL ratio between dogs affected or unaffected with cranial cruciate ligament rupture. Based on the results of this study we can conclude that the morphology of the cranioproximal tibia and the height of insertion point of the patellar ligament do not have an influence on the value of the modified IS index both in healthy dogs and in dogs with cranial cruciate ligament rupture.
\end{abstract}

Tibial tuberosity, patella alta/baja, canine stifle joint, cruciate disease

The most common orthopaedic disorders affecting the canine stifle joint and subsequently resulting in hind leg lameness are the cranial cruciate ligament rupture (CCLR) and medial, or lateral patellar luxation (Roush 1993; Johnson et al. 1994; Piermatei and Flo 1997; Necas and Zatloukal 2002; Towle et al. 2005). Although generally occurring as a separate problem, CCLR can occur in conjunction with medial or lateral patellar luxation (Hulse 2011). Diagnosis and treatment of these conditions consist of the measurement and surgical modification of tuberositas tibiae (Mortari et al. 2009). Also the location of the patella within the trochlear groove plays an important role in canine stifle joint stability. Proximodistal misalignment of the patella includes patella alta and baja, which refer to a patella located too proximally or distally within the femoral trochlea (Mostafa et al. 2008). Among the radiographic techniques described to evaluate the vertical position of the patella in humans, Insall Salvati (IS) index provides the most applicable and convenient method of diagnosing patella alta/baja in humans (Insall and Salvati 1971; Mostafa et al. 2008). The IS index is obtained by dividing the greatest diagonal length of the patella by the length of the patellar ligament to calculate a ratio, which is normally equal to 1.0. An IS index value $<0.80$ is consistent with patella alta, whereas a ratio $>1.20$ indicates the presence of patella baja (Insall and Salvati 1971; Carson et al. 1984; Simmons and Cameron 1992).

Modified IS index is obtained by dividing the distance between the inferior aspect of the articular surface of the patella and the insertion of the patellar ligament by the length of patellar articular surface. In spite of this modification, the clinical utility of IS index and

Address for correspondence:

MVDr. Dávid Kn̆azovický

Department of Surgery, Orthopaedics, Radiology and Reproduction

Small Animal Clinic

University of Veterinary Medicine and Pharmacy in Kosice

Komenskeho 73, 04081 Kosice, Slovak Republic

Phone: +421944393770

Email: knazovicky@uvm.sk

http://actavet.vfu.cz/ 
modified IS index remains limited by the difficulty in precisely determining the point of insertion of the patellar ligament on the tibial tuberosity (Mostafa et al. 2008).

The significance and clinical signs associated with patella baja or alta in dogs are less clear. In veterinary literature, patella alta is more frequently described than patella baja (Johnson et al. 2006; Mostafa et al. 2008)

Most studies in humans and dogs have focused on the diagnosis of patella alta and baja using techniques relying on the length of the patellar ligament, but have ignored the potential effects of distal femoral or proximal tibial abnormalities (Mostafa et al. 2008).

Therefore, the aim of this study was to assess whether the modified IS method can be used reliably in a canine patient despite differences of proximal tibial morphology and thus the insertion point of the patellar ligament. We aimed to determine a possible influence of the differences in cranio-proximal morphology of the tibia on the modified IS ratio in dogs with healthy stifles, and to determine if such differences are also seen in dogs with CCLR.

\section{Materials and Methods}

Medical and radiographic records of medium to large-breed dogs admitted between January 2006 and February 2011 were retrieved. Inclusion criteria were: skeletally mature (over 1 year of age), no history of trauma, no previous surgery of the stifle and no evidence of pathologic changes other than CCLR. All dogs were sedated (xylazine $1 \mathrm{mg} / \mathrm{kg}$ i.m. and butorphanol $0.2 \mathrm{mg} / \mathrm{kg}$ i.m.) for radiographic examination. Radiographs were assigned to two groups: healthy dogs and dogs with known CCLR as determined by intra-operative findings or the presence of cranial drawer and positive tibial compression test on orthopaedic examination. We evaluated medio-lateral radiographs of stifle joints, where stifle and tarsal joints were positioned at $90^{\circ}$ angles in respect to each other. Inclusion criteria for radiographs were superimposition of the femoral condyles, or separated caudally, distally or cranially less than $2 \mathrm{~mm}$. Radiographs were digitized with a digital camera Panasonic DMC-T27 (Panasonic Corporation, Japan).

The patellar ligament length (PLL) was measured on the cranial aspect of the ligament, from the point of the origin of the ligament on the distal aspect of the patella to its insertion on the most cranio-proximal extent of the tibial tuberosity (Osmond et al. 2006; Mostafa et al. 2008) (Plate III, Fig. 2). The length of the patella (PL) was determined by measurement of the longest dimension of the patella (Johnson et al. 2006). The PLL:PL ratio was calculated using equation PLL/PL. All measurements were determined using image measurement software Digimizer Version 4.1.1.0 (MedCalcSoftware, www.digimizer.com).

Position of the tibial insertion point of the patellar ligament was determined as follows: the length of the tibia was divided into 64 sections (Plate III, Fig. 3). We choose 64 from this equation: $2 \times 2 \times 2 \times 2 \times 2 \times 2=64,2$ is a factor 6 times, in other words we divided the tibial functional axis 6 times, always in half. By this calculation we standardized the length of the tibia for all dogs independent of the body weight. To the authors' knowledge, this method of determining the height of the insertion point of patellar ligament has not been previously reported. The length of the tibia was measured on the tibial functional axis as a distance between the most proximal point on the tibia between the intercondylar tubercles and the point of contact of the distal tibia and talocrural joint, the most proximal part of the half-arch. Perpendicular lines to the tibial functional axis were drawn over the proximal part of the tibia with distance between them equal to 1/64 part of the length of the tibia. The position of the insertion point of the cranial aspect of the straight patellar ligament was recorded (Point A in Fig. 3)

All data were included into a spreadsheet program (Microsoft Office Excel 2007, Microsoft Corporation) and imported into statistical software (GraphPad Prism 5, GraphPad Software, Incorporated) for analysis. A linear regression analysis was calculated for the IS

Table 1. Mean $( \pm \mathrm{SD})$ values for radiographic measurements and ratio of patellar ligament length to patellar length for healthy dogs and dogs with cranial cruciate ligament rupture

\begin{tabular}{lcc}
\hline Measurement $(\mathrm{cm})$ & $\begin{array}{c}\text { Healthy dogs } \\
(\mathrm{n}=25)\end{array}$ & $\begin{array}{c}\text { Dogs with CCLR } \\
(\mathrm{n}=26)\end{array}$ \\
\hline PL & $2.28 \pm 0.27$ & $2.19 \pm 0.42$ \\
PLL & $5.16 \pm 0.58$ & $4.84 \pm 1.20$ \\
PLL/PL & $2.27 \pm 0.25$ & $2.18 \pm 0.29$ \\
\hline
\end{tabular}

PL - patellar length, PLL - patellar ligament length, PLL/PL - patellar ligament length to patellar length, CCLR - cranial cruciate ligament rupture No significant difference between values for the PPL/PL for healthy and CCLR dogs $(P=0.07)$. index and the value of the point A. Student's $t$-test was used to compare the IS index between groups of healthy dogs and RCCL dogs. Significance was set at $P<0.05$.

\section{Results}

One-hundred and two stifles met the criteria for inclusion. Fifty-one dogs with both stifles underwent the evaluation of which twenty-five $(49 \%)$ dogs were clinically healthy with no radiographic evidence of orthopaedic disease and twenty-six 


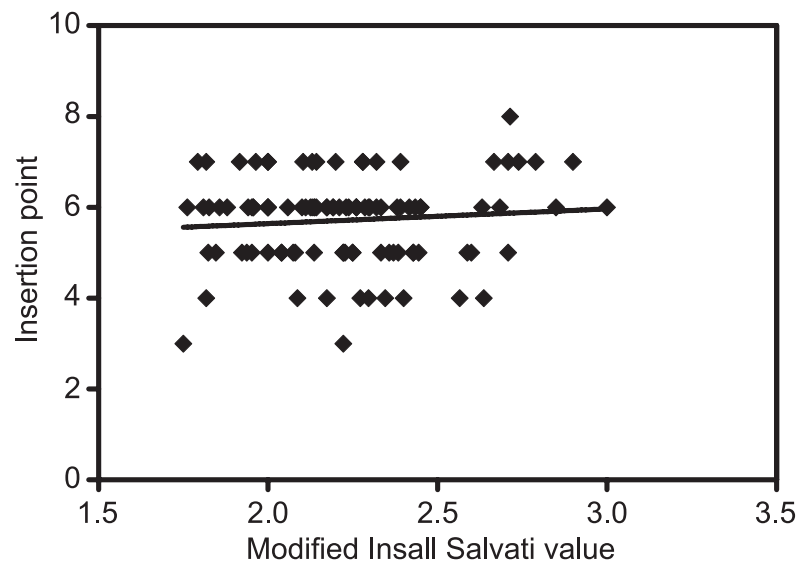

Fig 1. Magnitude of the Insall Salvati index and the point of insertion of patellar ligament on tibia (X/64).

$(51 \%)$ dogs were affected unilaterally by CCLR. The mean ( \pm SD) body weight was 37.3 $\pm 14.1 \mathrm{~kg}$ (range $12-82 \mathrm{~kg}$ ) and the mean age was $4.3 \pm 3.2$ years (range $1-12$ years). The mean modified IS index was $2.23 \pm 0.27$ (range 1.75-3.0) for all dogs; for healthy dogs it was $2.27 \pm 0.25$ (range 1.79-3.0) and for dogs with CCLR it was $2.18 \pm 0.29$ (range 1.75-2.9) (Table 1). Position of point A was at: 3/64 (2 stifles), 4/64 (9 stifles), 5/64 (26 stifles), 6/64 (45 stifles), 7/64 (19 stifles) and 8/64 (1 stifle). Measurements of the length of the patella, patellar ligament and patellar ligament/patella ratio are given in Table 1 . There was no significant correlation $(P=0.36)$ between the point of insertion of the patellar ligament and the value of modified IS index (Fig. 1). There was also no significance $(P=0.07)$ of the modified IS index between groups of healthy and CCLR dogs.

\section{Discussion}

The patella is an important structure that enhances the mechanical efficiency of the quadriceps muscle and helps maintain stifle stability (Payne and Constantinescu 1993). Stifle stability is compromised when the patella slips out of its normal position. This may predispose the dog to ligament injury, in particular the cranial cruciate ligament, which can lead to the progression of arthritis. Concurrent patellar luxation and cranial cruciate rupture is not a rare combination of injuries and may occur in up to $40 \%$ of dogs with patellar luxation (Campbell et al. 2010). Pathological vertical positions of the patella include patella alta and patella baja, which might predispose dogs to patellar luxation. To define the vertical position in dogs, a modified IS method has been described (Mostafa et al. 2008). The method is defined as a ratio of the length of the patellar ligament to the length of the patella. Identification of the landmarks may be difficult with the presence of degenerative joint disease. Osteophytes on the distal aspect of the patella make it difficult to determine the length of the normal patella. The observer must estimate where the normal distal margin of the patella ends and where the osteophyte begins. Prominent osteophytes at the distal aspect of the patella make this determination difficult and less precise. The point of insertion of the patellar ligament is associated with a small indentation on the tibial tubercle which can be used as a landmark to measure the patellar ligament in dogs (Johnson et al. 2006; Mostafa et al. 2008). Johnson et al. (2002) were assuming that the tibial insertion is constant, although from clinical observations, there is variability of 
the cranio-proximal part of the tibia, and the insertion point of the patellar ligament is not at a constant height on the tibial tubercle. Our measurement (1.75-3.0) of the modified IS index shows higher values compared to 1.45-2.17 and 1.77-2.29 obtained in the studies of Johnson et al. (2006) and Mostafa et al. (2008), respectively. The PLL:PL values $>1.97$ (Johnson et al. 2006) and > 2.06 (Mostafa et al. 2008) were consistent with patella alta and might have association with medial patellar luxation. It is important to note that we did not diagnose medial or lateral patellar luxation. We measured the cranial aspect of the patellar ligament, which may have increased the length of the patellar ligament, reflecting in our higher values of the modified IS ratio. If we measured the length of patellar ligament on its caudal aspect as Johns on et al. (2006) did, we might have got a lower IS ratio value. Values for modified IS ratio were previously reported to be independent of the stifle angle (Johnson et al. 2002). We choose the $90^{\circ}$ angle based on Mostafa et al. (2008) because at this angle the modified IS ratio was at its maximal value.

There is a relationship between the shape of the tibia and pathogenesis of CCLR (Osmond et al. 2006). Most of the discussion is focused on the relationship of the tibial plateau slope and CCLR (Morris and Lipowitz 2001; Reif and Probst 2003; Zeltman et al. 2005).

Our study has several limitations. The size of our sample was small. We randomly chose a dog to prepare our model without knowing, whether or not its tibia would represent normal or average tibia. Another limitation was the evaluation of the radiographs. All radiographs were evaluated by one observer in order to avoid inter-observer variability. We recommend more observers to avoid possible errors in measurements caused by one observer.

.To further define the influence of bone morphology on specific orthopaedic diseases we recommend evaluation of tibial morphology in larger population of dogs. We can state that cranio-proximal variations of the tibia do not influence CCLR or medial or lateral patellar luxation. The vertical patellar position can be assessed using the modified IS method but larger population of dogs must be evaluated for the unification of the measurement results.

\section{Acknowledgements}

This study was carried out with the financial support from the Scientific Grant Agency of the Ministry of Education of the Slovak republic and the Slovak Academy of Sciences (VEGA) 1/0631/09

\section{References}

Campbell CA, Horstman CL, Mason DR, Evans RB 2010: Severity of patellar luxation and frequency of concomitant cranial cruciate ligament rupture in dogs: 162 cases (2004-2007). J Am Vet Med Assoc 236: 887-891

Carson WG, James SL, Larson LL, Singer KM, Winternitz WW 1984: Patellofemoral disorders. Physical and radiographic evaluation. Clin Orthop Rel Res 185: 178-186

Hulse D 2011: Concomitant CCL and MPL repair with the arthrex stabilisation system. In: ESVOT proceedings 2011: Seminar on patellar luxation. Lyon, p 37

Insall J, Salvati E 1971: Patellar position in the normal knee joint. Diagnostic Radiol 101: 101-104

Johnson JA, Austin C, Breur GJ 1994: Incidence of canine appendicular musculoskeletal disorders in 16 veterinary teaching hospitals from 1980 through 1989. Vet Comp Orthop Traumatol 7: 56-69

Johnson AL, Broaddus KD, Hauptman JG, Marsh S, Monsere J, Sepulveda G 2006: Vertical patellar position in large-breed dogs with clinically normal stifles and large-breed dogs with medial patellar luxation. Vet Surg 35: $78-81$

Johnson AL, Probst CW, DeCamp CE, Rosenstein DS, Hauptman JG, Kern TL 2002: Vertical position of the patella in the stifle joint of clinically normal large-breed dogs. Am J Vet Res 63: 42-46

Morris E, Lipowitz AJ 2001: Comparison of tibial plateau angles in dogs with and without cranial cruciate ligament injuries. J Am Vet Med Assoc 218: 363-366

Mortari AC, Rahal SC, Vulcano LC, Colombi da Silva V, Volpi RS 2009: Use of radiographic measurements in the evaluation of dogs with medial patellar luxation. Can Vet J 50: 1064-1068

Mostafa AA, Griffon DJ, Thomas MW, Constable PD 2008: Proximodistal alignment of the canine patella: radiographic evaluation and association with medial and lateral patellar luxation. Vet Surg 37: 201-211 
Necas A, Zatloukal J 2002: Factors related to the risk of meniscal injury in dogs with cranial cruciateligament rupture. Acta Vet Brno 71: 77-84

Osmond CS, Marcellin-Little DJ, Harrysson OLA, Kidd LB 2006: Morphometric assessment of the proximal portion of the tibia in dogs with and without cranial cruciate ligament rupture. Vet Radiol Ultrasound 47: 136-141

Payne JT, Constantinescu GM 1993: Stifle joint anatomy and surgical approaches in the dog. Vet Clin North Am Small Anim Pract 23: 691-701

Piermattei DL, Flo GL 1997: The stifle joint. In: Piermattei DL, Flo GL (Ed.): Handbook of small animal orthopedics and fracture repair (Ed 3). Saunders, Philadelphia, pp. 516-534

Reif U, Probst CW 2003: Comparison of tibial plateau angles in normal and cranial cruciate deficient stifles of Labrador retrievers. Vet Surg 32: 385-389

Roush JK 1993: Canine patellar luxation. Vet Clin North Am Small Anim Pract 23: 855-868

Simmons E, Cameron JC 1992: Patella alta and recurrent dislocation of the patella. Clin Orthop Rel Res 274: 265-269

Towle HA, Griffon DJ, Thomas MW, Siegel AM, Dunning D, Johnson A 2005: Pre- and postoperative radiographic and computed tomographic evaluation of dogs with medial patellar luxation. Vet Surg 34: 265-272

Zeltzman PA, Pare B, Johnson GM, Zeltzman V, Robbins MA, Gendreau CL 2005: Relationship between age and tibial plateau angle in dogs with cranial cruciate rupture. J Am Anim Hosp Assoc 41: 117-120 
Kňazovický D. et al.: Use of the modified ...pp. 403-407

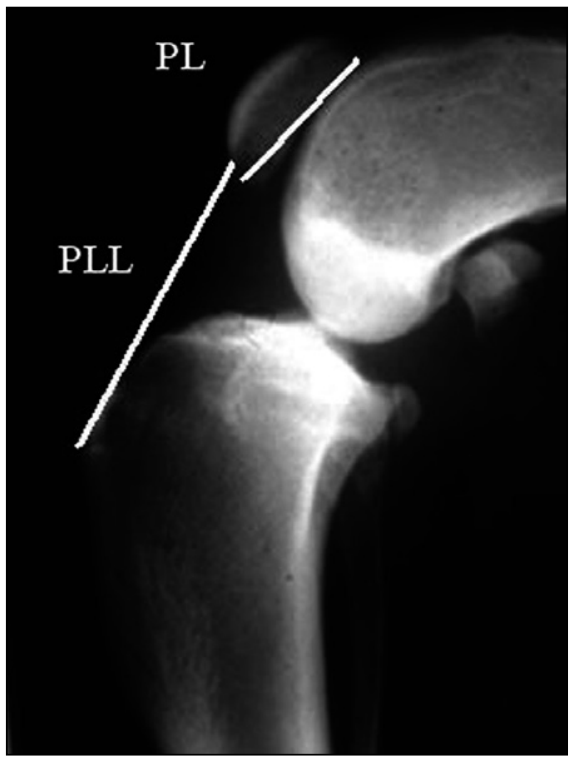

Fig 2. Medio-lateral radiograph of a canine stifle showing measurements for determination of patellar ligament length (PLL) and patellar length (PL).

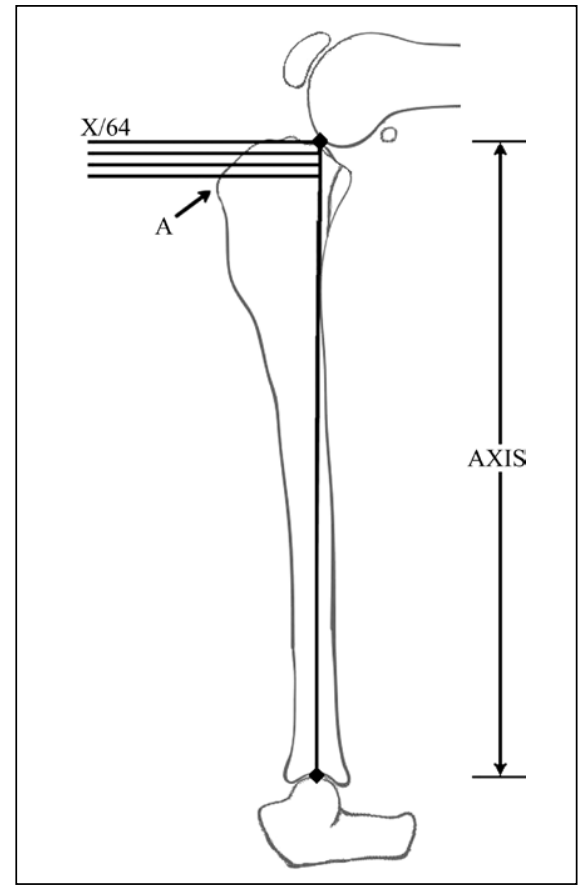

Fig 3. Schematic illustration of canine tibia in a medio-lateral view, showing the method to measure the height if insertion point $(\mathrm{A})$ 